\title{
Decoding Turbo-Like Codes via Linear Programming
}

\author{
Jon Feldman David R. Karger*
}

\author{
MIT Laboratory for Computer Science \\ Cambridge, MA, 02139 \\ \{jonfeld,karger\}@theory.lcs.mit.edu
}

\begin{abstract}
We introduce a novel algorithm for decoding turbo-like codes based on linear programming. We prove that for the case of Repeat-Accumulate (RA) codes, under the binary symmetric channel with a certain constant threshold bound on the noise, the error probability of our algorithm is bounded by an inverse polynomial in the code length.

Our linear program $(L P)$ minimizes the distance between the received bits and binary variables representing the code bits. Our LP is based on a representation of the code where code words are paths through a graph. Consequently, the $L P$ bears a strong resemblance to the min-cost flow LP. The error bounds are based on an analysis of the probability, over the random noise of the channel, that the optimum solution to the LP is the path corresponding to the original transmitted code word.
\end{abstract}

\section{Introduction}

The introduction of turbo codes [3] revolutionized the field of coding theory by achieving an error probability orders of magnitude smaller than any other code at the time. Since then, volumes of research has focused on design, implementation, and analysis of turbo codes and their variants and generalizations [17].

One of the main goals of this research has been to explain the somewhat mysterious good performance of turbo codes and turbo-like codes. Even though the distances of turbolike codes are generally bad $[10,2,5]$, when decoded using an iterative decoder, they seem to achieve very good error rates [6]. The drawback to an iterative decoder is that it not

\footnotetext{
* Research supported by NSF contract CCR-9624239 and a David and Lucille Packard Foundation Fellowship.
}

guaranteed to converge, nor does it have any guarantee on the quality of its output.

Some progress has been made assuming optimal maximum-likelihood (ML) decoding, for which no polynomial-time algorithm is known. It is known [6] that if the noise in the channel is under a certain constant threshold, ML decoding of randomly generated turbo codes has an error probability bounded by an inverse polynomial in the code length, as the code length goes to infinity. The first such result by Divsalar and McEliece[6] was for a type of turbo-like code called a repeat-accumulate (RA) code.

There are several drawbacks to these results. The fact that they do not apply to a specific constructible code forces the designer of the code to choose a random one, and thus be uncertain about its quality. Additionally, in many cases the asymptotic nature of the bound requires a large block length, whereas small fixed code lengths are desirable in practice to reduce latency in transmission. Most importantly, however, the given error probability is proven only under ML decoding, for which no efficient algorithm is known.

Our Results. In this paper we introduce a novel approach to decoding any turbo-like ${ }^{1}$ code based on linear programming. We prove that for the case of Repeat-Accumulate (RA) codes, with a certain constant threshold bound on the noise in the channel, the error probability of our algorithm is bounded by an inverse polynomial in the code length. We improve upon previous results in three important respects. Our analysis holds for (i) a provably polynomialtime decoding algorithm, (ii) a specific, deterministically constructible code, and (iii), any code length.

More precisely, we show that for a particular RA code

\footnotetext{
${ }^{1}$ We note that our definition for turbo-like codes is along the lines of [6], where the class includes any serial or parallel concatenated convolutional code.
} 
with rate $1 / 2-o(1)$ and length $n$, our LP makes an error with probability at most $n^{-\epsilon}$, for any $\epsilon>0$, as long as $p<2^{-4(\epsilon+(\log 24) / 2)}$, where each code bit is flipped by the channel with probability $p$. As $\epsilon \rightarrow 0$, the threshold on $p$ approaches $\approx 2^{-9.17}$. For lower rate RA codes, our bound on error rate is trivially applicable by simply decoding an embedded rate-1/2 RA code; however, we expect that a more general analysis will yield better bounds.

When applied to any turbo-like code, our decoder has the desirable property that when it outputs a code word, it is guaranteed to be the maximum-likelihood code word. As far as the authors are aware, no other efficient algorithm for decoding turbo-like codes is known to have this $M L$ certificate property. Additionally, the key structural theorem used to prove the error bound for RA codes easily generalizes to other turbo-like codes, and provides a good basis for proving better error bounds.

Previous Work. A breakthrough in the analysis of turbo codes came when McEliece, MacKay and Cheng[12] showed how the classic iterative decoding method for turbo codes is an instance of Pearl's belief propagation (BP) algorithm, a standard tool used in the artificial intelligence community. Now most work in the areas of turbo codes and low-density parity check (LDPC) codes is interpreted in this context (Richardson, Shokrollahi and Urbanke[13], for example).

The convergence of BP algorithms becomes difficult to prove when the underlying "belief network" contains cycles, as is the case for turbo codes. However, a lot of progress has been made by analyzing average codes (or "code ensembles"), giving various tradeoffs involving rate, probability thresholds, and iterations of the BP algorithm [14].

In follow-up work (with Wainwright [8]), we have shown that the the recent iterative tree-reweighted max-product (TRMP) algorithm for MAP estimation of graphical models of Wainwright, Jaakkola and Willsky [18], when applied to the problem of decoding turbo-like codes, has a fixed point equivalent to the solution of the LP we present here. Thus we have begun to connect our LP-based decoder with the world of iterative decoders.

The minimum distance of a code is the minimum Hamming distance between any two code words. The minimum distance of turbo-like codes has received some attention recently $[10,2,5]$. Most of the work has focused on the negative side, showing that the minimum distance of a turbolike code is sub-linear. Kahale and Urbanke [10] give highprobability upper and lower bounds on the minimum distance of a random interleaver, as the block length goes to infinity, for any parallel or serially concatenated convolutional code. Bazzi, Mahdian, Miller and Spielman [2] give similar upper bounds over all interleavers for some of the same types of codes. They also give a construction of a rate$1 / 2$ RA code and show its minimum distance is $\Omega(\log n)$. We will discuss this code in more detail later in the paper.

Coding Theory Background. An error-correcting code is used to build redundancy into a data stream for transmission over an unreliable channel. The sending party takes a binary vector $x$ of length $k$ (the information word), applies an encoder (a function $E:\{0,1\}^{k} \rightarrow\{0,1\}^{n}$ ), to obtain a code word of $n$ bits, $n \geqslant k$ (the parameter $n$ is referred to as the code length or block length), and transmits the code word $y=E(x)$ over the channel. The rate of the code is $k / n$. The code word is then subject to an unreliable channel that can be modeled in several ways. Here we will use the binary symmetric channel (BSC), where each bit of the code word is flipped independently with probability $p$ (called the crossover probability). Our results in this paper also hold for the additive white Gaussian noise (AWGN) channel, where the channel adds an independent random Gaussian variable to each transmitted bit; for clarity, we discuss only the BSC.

The receiving party gets the corrupted code word $v$ of length $n$, and must try to recover the original information word $x$ using a decoder. The decoder is simply a function $D:\{0,1\}^{n} \rightarrow\{0,1\}^{k}$. The word error probability $P_{w}$ of the decoder is the probability, over the random coin flips of the channel, that $D(v) \neq x$. The maximum-likelihood (ML) information word $x$ is the one that maximizes $\operatorname{Pr}[E(x)$ was sent $\mid v$ was received ]. Using Bayes' rule, and the conventional assumption that all information words have equal probability, this is the same information word $x$ that maximizes $\operatorname{Pr}[v$ was received $\mid E(x)$ was sent $]$. Thus, under the BSC, $x$ is the information word that minimizes the Hamming distance between $D(x)$ and $v$. Note that the ML information word is not necessarily the original encoded information word. An ML decoder is one that always finds the ML information word.

The purpose of an error-correcting code is to be robust against noise, so the word error probability $P_{w}$ is the metric that should be used to measure the quality of a code. Codes are often measured for quality in terms of their minimum distance $d$. This is the minimum, over all pairs of valid code words, of the Hamming distance between the pair. It is not hard to see that an ML decoder will always correct up to $\lceil d / 2\rceil-1$ errors in the channel, so a large minimum distance is desirable. However, the minimum distance is a "worst-case" measure, so considering it as the only measure of quality ignores other important attributes of the code that affect the word error probability. In fact, turbo codes are a perfect example of codes whose minimum distance is considered bad, but whose word error probability is good.

We will use the definitions given here for $k, n, x, y, v$ and $p$ throughout the paper. For more background on error- 
correcting codes, we refer the reader to textbooks written on the subject $[19,11]$.

Our Techniques. We show that the problem of finding the ML code word (referred to as ML decoding) can be solved by finding an optimal integral point inside a polytope with linear constraints. Binary variables represent the information word $x$, and the objective function is to minimize $\operatorname{Pr}[v$ was received $\mid x$ was sent $]$, where $v$ is the corrupted code word received from the channel. We relax the integral constraints to obtain a linear program (LP). The algorithm solves the LP, and if the solution is integral, outputs the information word, which it knows is the ML information word. If the solution is not integral, the algorithm outputs "error." This approach guarantees the ML-certificate property discussed earlier.

In the setting of decoding algorithms, the rounding scheme for an LP relaxation is not as important as it is in other more conventional optimization problems. Because the LP is only guaranteed to output the ML information word if it finds an integral solution, even if we provide a rounding scheme with a provably small approximation ratio, it does not help bound the probability that the decoder returns the ML information word.

Therefore, instead of analyzing the approximation ratio as is normally done, we assume that the algorithm is always wrong when the solution is fractional, and bound the probability that the LP returns the real information word that was transmitted (not the ML word). By doing so, not only do we bound the error probability of our polynomial-time algorithm, we also bound the error probability of ML decoding; this is because whenever our LP finds the correct solution, this solution must also be the optimal point in the integral polytope, and an ML decoder would have found it as well.

Our LP can be used to decode any set of concatenated codes, with interleavers between them. However, the LP has polynomial size only if the component codes can be expressed using a polynomial-sized "trellis." The simplest such codes are the class of convolutional codes (see [19]). Thus we say that the LP decoder is a decoder intended for "turbo-like" [6] codes: serial or parallel concatenated convolutional codes.

Outline. In Section 2, we define repeat-accumulate (RA) codes, and present our linear program as applied to RA codes. In Section 3 we state our main structural theorem (proven in Section 4), and show our bound on the probability that the LP returns the original information word. We discuss the generalization of the linear program to any set of concatenated codes in Section 5. In Section 6 we give several interesting open questions that arise from this work.

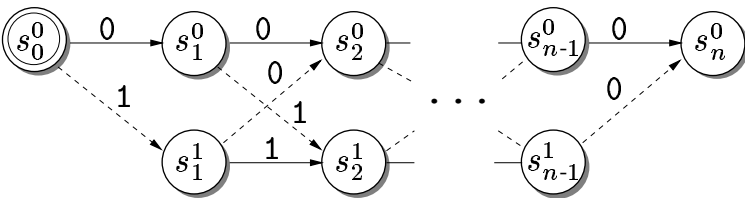

$\begin{array}{lll}\text { Seg. } 0 & \text { Seg. } 1 & \text { Seg. } n-1\end{array}$

Figure 1. The trellis for an accumulator, used in RA codes. The dashed-line switch-edges correspond to information bit 1, the solid-line remain-edges to information bit 0 . The edges are labeled with their associated output code bit.

\section{Repeat-Accumulate Codes}

Repeat-Accumulate codes are perhaps the simplest nontrivial example of a turbo-like code. They were introduced by Divsalar and McEliece [6] in order to show the first bounds for error probabilities under ML decoding. Their simple structure and highly efficient encoding scheme make them both practical and simpler to analyze than other more complex turbo-like codes. They have also been shown experimentally to have excellent error-correcting ability under iterative decoding [6], on par with classic turbo codes.

Encoding. The encoder for an RA code takes the input word, repeats every bit $\ell$ times, then sends it through an interleaver (known permutation), and then through an accumulator. The accumulator maintains a partial sum mod 2 of the input seen so far, and outputs the new sum at each step.

More formally, an $\mathrm{RA}(\ell)$ code of length $n=\ell k$ has an interleaver (permutation) $\pi:\{0, \ldots, n-1\} \rightarrow\{0, \ldots, n-$ $1\}$. The encoder is given an information word $x$ of length $k$. Let $x^{\prime}$ be the length- $n$ repeated and permuted information word, i.e., for all $t \in\{0, \ldots, n-1\}, x_{t}^{\prime}=x_{\left\lfloor\pi^{-1}(t) / \ell\right\rfloor}$. The RA encoder outputs a code word $y$ of length $n$, where for all $j \in\{0, \ldots, n-1\}, y_{j}=\sum_{t=0}^{j} x_{t}^{\prime} \bmod 2$.

For all $i \in\{0, \ldots, k-1\}$, let $X_{i}$ be the set of indices to which information bit $x_{i}$ was repeated and permuted, i.e., $X_{i}=\{\pi(\ell i), \pi(\ell i+1), \ldots, \pi(\ell i+\ell-1)\}$. Let $\mathcal{X}=$ $\left\{X_{i}: i \in\{0, \ldots, k-1\}\right\}$. To keep the proofs in this paper simpler, we assume that the input contains an even number of $1 \mathrm{~s}$. This can be achieved by padding the information sequence with an extra parity bit. Thus the rate of this code is $(k-1) / \ell k$, or $1 / \ell-o(1)$. The $o(1)$ can be avoided by a more technical proof, which we leave out for clarity.

The Accumulator Trellis. A trellis is a simple layered graph that models the actions of a finite-state encoder over time, as it encodes a binary string passed to it. The trellis is the basis for the classic Viterbi decoding algorithm $[16,9]$. 
Figure 1 shows the trellis $T$ for an accumulator.

To derive the trellis, we view the accumulator as a simple finite state machine, receiving the $n$-bit binary input string $x^{\prime}$ one bit at a time. The accumulator has two possible states per time step, depending on the parity of the input bits seen so far. We refer to the two states of the accumulator as 0 and 1 ; state 0 represents even parity, 1 represents odd parity. We use $\left\{s_{0}^{0}, \ldots, s_{n}^{0}\right\}$ and $\left\{s_{1}^{1}, \ldots, s_{n-1}^{1}\right\}$ to refer to the sets of even and odd parity nodes, respectively.

An encoder using this trellis begins in state $s_{0}^{0}$. At each time step, if it receives a 1 as input, it follows the dashedline transition to the next layer of nodes, switching states. We call this a switch-edge. If it receives a 0 , it follows the solid-line transition to the next layer of nodes, staying in the same state (see Figure 1). We call this type of edge a remain-edge. The labels on the transition edges represent the code bit output by the encoder taking that transition; label 0 for edges entering state 0 , label 1 for edges entering state 1 .

A path from $s_{0}^{0}$ across the trellis to $s_{n}^{0}$ corresponds to an entire $n$-bit input string. Since the accumulator begins and ends in state 0 (the input string has even parity by assumption), we do not need the states $s_{0}^{1}$ and $s_{n}^{1}$. Looking at the edge labels on the path, one can read off the code word corresponding to that input string. Let $P_{x}$ be the path taken by the encoder through the trellis $T$ while encoding $x^{\prime}$.

We will refer to the group of four edges at one time step of the trellis as a segment. Define the cost $c[e]$ of an edge $e$ in the trellis at segment $t$ to be the Hamming distance between its label and the received bit $v_{t}$. The cost of a path is the sum of the costs of the edges on the path.

Decoding with the Trellis. Assume for the moment that the accumulator was the entire encoder (i.e., the information bits were fed directly into the accumulator without repeating and permuting). Then, all paths through the trellis from $s_{0}^{0}$ to $s_{n}^{0}$ represent valid information words, and the labels along the path represent valid code words. Furthermore, the cost of the path is the Hamming distance between the code word and the received word. A simple shortest-path computation thus yields the ML information word. The decoding algorithm we just described is exactly the Viterbi algorithm $[16,9,19]$.

However, if we tried to apply the Viterbi algorithm to RA codes, we would run into problems. For example, suppose $\ell=2$, and let $x_{i}=1$ be some arbitrary information bit, where $X_{i}=\{t, \hat{t}\}$. Since $x_{i}$ is input into the accumulator at time $t$ and time $\hat{t}$, any path through the trellis $T$ that represents a valid encoding would use a switch-edge at time step $t$, and at time step $\hat{t}$. In general, any path representing a valid encoding would do the same thing (as in switch levels or remain on the same level) at every time step $t \in X_{i}$. We say a path is agreeable for $x_{i}$ if it has this property for $x_{i}$.
An agreeable path is a path that is agreeable for all $x_{i}$. Any path that is not agreeable does not represent a valid encoding, and thus finding the lowest cost path is not guaranteed to return a valid encoder path.

What we would like to find is the lowest cost agreeable path from $s_{0}^{0}$ to $s_{n}^{0}$. We give a simple integer program based on min-cost flow that solves this problem.

RALP: Repeat-Accumulate Linear Program. For each node $s$ in the trellis, define $\delta(s)$ to be the set of outgoing edges from $s$, and $\gamma(s)$ to be the set of incoming edges. Our integer program contains variables $f(e) \in\{0,1\}$ for every edge $e$ in the trellis, and free variables $z_{i}$ for every information bit $x_{i}, i \in\{0, \ldots, n-1\}$. The relaxation RALP of the integer program simply relaxes the flow variables such that $0 \leqslant f(e) \leqslant 1$. RALP is defined as follows:

$$
\begin{aligned}
& \text { RALP: } \min \sum_{e \in T} c[e] f(e) \quad \text { s.t. } \\
& \sum_{e \in \gamma\left(s_{n}^{0}\right)} f(e)=1 \\
& \sum_{e \in \gamma(s)} f(e)=\sum_{e \in \delta(s)} f(e) \quad \forall s \in T \backslash\left\{s_{0}^{0}, s_{n}^{0}\right\} \\
& f\left(s_{t}^{0}, s_{t+1}^{1}\right)+f\left(s_{t}^{1}, s_{t+1}^{0}\right)=z_{i} \quad \forall X_{i} \in \mathcal{X}, t \in X_{i} \\
& 0 \leqslant f(e) \leqslant 1 \quad \forall e \in T
\end{aligned}
$$

RALP is very close to being a simple min-cost flow LP: Equation (1) gives a demand of one unit of flow at the sink node $s_{n}^{0}$, and equation (2) is a flow conservation constraint at each node. Unique to RALP are the agreeability constraints (3). These constraints say that a feasible flow must have, for all $X_{i} \in \mathcal{X}$, the same amount $z_{i}$ of total flow on switch-edges at every segment $t \in X_{i}$. Note that these constraints also imply a total flow of $1-z_{i}$ on remain-edges at every segment $t \in X_{i}$. We will refer to the flow values $f$ of a feasible solution $(f, z)$ to RALP as an agreeable flow. The free variables $z_{i}$ do not play a role in the objective function, but rather enforce constraints among the flow values.

Using RALP as a decoder. A decoding algorithm based on RALP is as follows. Run an LP-solver to find the optimal solution $\left(f^{*}, z\right)$ to RALP, setting the costs on the edges according to the received word $v$. If $f^{*}$ is integral, output $z$ as the decoded information word $x$. If not, output "error." We will refer to this algorithm as the RALP decoder.

All integral solutions to RALP represent agreeable paths, and thus valid encodings of some information word. This implies that if the optimal solution $f^{*}$ to RALP is in fact integral, then $f^{*}$ is the lowest cost agreeable path, and $z$ represents the ML code word. Thus the RALP decoder has the 
$M L$ certificate property: whenever it outputs an information word, it is guaranteed to be the ML information word. No standard iterative decoding techniques are known to have this property.

\section{A Coding Theorem for RA(2) Codes}

In this section we state our main structural theorem (proven in section 4). We then show how this theorem suggests a design for an interleaver for RA(2) codes, and prove an inverse polynomial upper bound on the RALP decoder's word error probability when we use this interleaver.

Our main structural theorem states that the RALP decoder succeeds (returns the original information word) if and only if a particular graph does not contain a certain negative-cost subgraph, generalizing the role of a negativecost cycle in min-cost flow. The graph has a structure that depends on the interleaver $\pi$ and weights that depend on the errors made by the channel. We note that an analogous theorem holds for any $\mathrm{RA}(\ell)$ code, or any turbo-like code for that matter. This is discussed further in Section 5.

For the remainder of this section we deal exclusively with RA(2) codes. This means that each set $X_{i} \in \mathcal{X}$ has two elements, and the agreeability constraints may be expressed as, $\forall X_{i} \in \mathcal{X}, X_{i}=\{t, \hat{t}\}$,

$z_{i}=f\left(s_{t}^{0}, s_{t+1}^{1}\right)+f\left(s_{t}^{1}, s_{t+1}^{0}\right)=f\left(s_{\hat{t}}^{0}, s_{\hat{t}+1}^{1}\right)+f\left(s_{\hat{t}}^{1}, s_{\hat{t}+1}^{0}\right)$.

The Promenade. Let $G=\left(V_{G}, E_{G}\right)$ be a weighted undirected graph with $n$ nodes $\left(g_{0}, \ldots, g_{n-1}\right)$ connected in a line, where the cost $c\left[g_{t}, g_{t+1}\right]$ of edge $\left(g_{t}, g_{t+1}\right)$ is -1 if the $t^{t h}$ bit of the transmitted code word is flipped by the channel $\left(v_{t} \neq y_{t}\right)$, and +1 otherwise. Call these edges the Hamiltonian edges, since they make a Hamiltonian path. Note that these costs are not known to the decoder, since they depend on the transmitted code word. For each $\{t, \hat{t}\} \in \mathcal{X}$, add an edge between node $g_{t}$ and node $g_{\hat{t}}$ with cost 0 . Call these edges the matching edges. Note that $G$ is a line plus a matching.

Define a promenade to be a circuit in $G$ that begins and ends in the same node, and may repeat edges as long as it does not travel along the same edge twice in a row. The cost of a promenade is the total cost of the edges visited during the path, including repeats (i.e., repeats are not free). Formally, a promenade is a path $M=\left(p_{0}, p_{1}, \ldots, p_{|M|}=\right.$ $\left.p_{0}\right)$ in $G$ that begins and ends at the same node $p_{0}$, where for all $i \in\{0, \ldots,|M|-1\}, p_{i} \neq p_{i+2} \bmod |M|$. The cost of a promenade $M$ is $c[M]=\sum_{i=0}^{|M|-1} c\left[p_{i}, p_{i+1}\right]$. We are now ready to state our main structural theorem.

Theorem 1 The RALP decoder succeeds if all promenades in $G$ have positive cost. The RALP decoder fails if there is a promenade in $G$ with negative cost.
When there is a zero-cost promenade, the RALP decoder may or may not decode correctly (this is a degenerate case when the LP has multiple optima). We will prove Theorem 1 in Section 4, but first we show what it suggests about interleaver design, and how it can used to prove a bound on the probability of error of our algorithm.

Recall that every Hamiltonian edge of $G$ has cost -1 with some small constant probability $p$, so promenades with many edges are less likely to have a total negaitve cost (at least every other edge of a promenade is Hamiltonian). The girth of a graph is the length of its shortest simple cycle. It is not hard to see that every promenade contains at least one simple cycle, and so graphs with high girth will have promenades with many edges. This suggests that what we want out of an interleaver, if we are to use the RALP decoder, is one that produces a graph $G$ with high girth.

We use a result of Erdös and Sachs [7] and Sauer [15] (see also [4]) to make a graph that is a line plus a matching, and has high girth. Their construction allows us, in cubic time, to start with an $n$-node cycle and build a 3-regular graph $G$ with girth $\lfloor\log n\rfloor$ that contains the original cycle. We remove an edge from the original cycle to obtain a line plus a matching with girth at least as high. To derive the interleaver, we simply examine the edges added by the construction. We will refer to this interleaver as $\pi_{E}$. This is the same construction used by Bazzi et al. [2] to show a $1 / 2 \log n$ lower bound on the minimum distance of an RA code using this interleaver.

Theorem 2 [2] The rate 1/2 - o(1) RA code with block length $n$, using $\pi_{E}$ as an interleaver, has minimum distance of at least $\frac{1}{2} \log n$.

Error Bound. We would like to bound the probability that $G$ contains a promenade with cost less than or equal to zero, thus bounding the probability that the RALP decoder fails. However, there are many promenades in $G$, so even a tight bound on the probability that a particular one is negative is insufficient. Furthermore, the fact that promenades may repeat edges creates dependencies that interfere with using standard Chernoff bound analysis. Consequently, we need to find a simpler structure that is present when there is a promenade with cost less than or equal to zero. In the following, a simple path or cycle means a path or cycle that does not repeat edges. For clarity (to avoid floors and ceilings), we will assume $n$ is a power of 16 , though our arguments do not rely on this assumption.

Lemma 3 If $n \geqslant 2^{4}$, and there exists a promenade $M$ in $G, c[M] \leqslant 0$, then there exists a simple path or cycle $Y$ in $G, c[Y] \leqslant 0$, that contains $\frac{1}{2} \log n$ Hamiltonian edges.

Proof: Let $M=\left(p_{0}, p_{1}, \ldots, p_{|M|-1}, p_{|M|}=p_{0}\right)$ be a promenade in $G, c[M] \leqslant 0$. Since $M$ contains a cycle, 
$|M| \geqslant \log n$. Contract every matching edge in $M$ to obtain a tour $H=\left(h_{0}, h_{1}, \ldots, h_{|\boldsymbol{H}|}=h_{0}\right\}$. No two matching edges share an endpoint, so at worst every other edge of the tour is a matching edge. Thus, $|H| \geqslant \frac{1}{2}|M| \geqslant \frac{1}{2} \log n$. Let $c[H]=\sum_{i=0}^{|H|-1} c\left[h_{i}, h_{i+1}\right]$. We know that $h_{i} \neq h_{i+2}$, $0 \leqslant i<|H|$ (operations on indices of $h \in H$ are performed $\bmod |H|)$; if this was not the case, then there would be a cycle in $G$ of length 3, violating our assumption that $n \geqslant 2^{4}$, since the girth of $G$ is at least $\log n$. Let $H_{i}=\left(h_{i}, \ldots, h_{i+\frac{1}{2} \log n}\right)$ be the subsequence of $H$ whose edge set contains $\frac{1}{2} \log n$ edges, and let $c\left[H_{i}\right]=$ $\sum_{j=i}^{i+\frac{1}{2} \log n-1} c\left[h_{j}, h_{j+1}\right]$. We know that $H_{i}$ has no repeated edges (i.e., it is a simple path or a simple cycle); if it were not, then if we added the matching edges back into $H$ we would get a path in $G$ with at most $\log n$ edges that repeated an edge, which would imply a cycle in $G$ of length less than $\log n$.

Since all matching edges have zero cost, $c[M]=c[H]$. Note that $c[H]=\left(\sum_{i=0}^{|H|-1} c\left[H_{i}\right]\right) /\left(\frac{1}{2} \log n\right)$, since every edge of $H$ is counted in the sum exactly $\left(\frac{1}{2} \log n\right)$ times. Since $c[H]=c[M] \leqslant 0$, at least one simple path or cycle $H_{i^{*}}$ must have $c\left[H_{i^{*}}\right] \leqslant 0$. Set $Y$ to be the simple path or cycle in $G$ obtained by expanding the zero-cost matching edges in the path or cycle $H_{i^{*}}$.

Theorem 1, along with the above construction of $G$ and a union bound over the paths of length $\frac{1}{2} \log n$ of $G$, gives us an analytical bound on the probability of error when decoding with the RALP decoder:

Theorem 4 For any $\epsilon>0$, the rate $1 / 2-o(1) R A$ code with block length $n$ using $\pi_{E}$ as an interleaver decoded with the RALP decoder under the binary symmetric channel with crossover probability $p<2^{-4(\epsilon+(\log 24) / 2)}$ has a word error probability $P_{w}$ of at most $n^{-\epsilon}$.

Proof: By Theorem 1, and Lemma 3, the RALP decoder succeeds if all simple paths or cycles in $G$ with $\frac{1}{2} \log n$ Hamiltonian edges have positive cost. We claim that there are at most $n \cdot 3^{\frac{1}{2} \log n}$ different simple paths that have $\frac{1}{2} \log n$ Hamiltonian edges. To see this, consider building a path by choosing a node, and building a simple path from that node. At each point, there are at most three choices for the next Hamiltonian edge.

The remainder of the proof is a union bound over the paths of $G$ with $\frac{1}{2} \log n$ Hamiltonian edges. Let $Y$ be a particular path with $\frac{1}{2} \log n$ Hamiltonian edges. Each Hamiltonian edge has cost -1 or +1 , so at least half of the Hamiltonian edges must have cost -1 in order for $c[Y] \leqslant 0$. Each Hamiltonian edge had cost -1 with probability $p$, so the probability that $c[Y] \leqslant 0$ is at most $\left(\begin{array}{c}\frac{1}{2} \log n \\ \frac{1}{4} \log n\end{array}\right) p^{\frac{1}{4} \log n}$. By the union bound,

$$
\begin{aligned}
P_{w} & \leqslant 3^{\frac{1}{2} \log n}\left(\begin{array}{c}
\frac{1}{2} \log n \\
\frac{1}{4} \log n
\end{array}\right) p^{\frac{1}{4} \log n} \\
& \leqslant n^{1+\frac{1}{2} \log 3} n^{\frac{1}{2}} 2^{-\left(\epsilon+\frac{1}{2} \log 24\right) \log n} \\
& \leqslant n^{1+\frac{1}{2} \log 3+\frac{1}{2}-\left(\epsilon+\frac{1}{2} \log 24\right)} \\
& \leqslant n^{-\epsilon}
\end{aligned}
$$

\section{Proof of Theorem 1}

Theorem 1 The RALP decoder succeeds if all promenades in $G$ have positive cost. The RALP decoder fails if there is a promenade in $G$ with negative cost.

In this section we prove Theorem 1. We assume a working knowledge of the min-cost flow problem, specifically the notions of a residual graph, a circulation and a path decomposition [1].

Let $f^{0}$ be the unit flow along the path $P_{x}$ taken by the encoder. The RALP decoder will succeed if $f^{0}$ is the unique optimal solution to RALP. The RALP decoder will fail if $f^{0}$ is not an optimal solution to RALP. To prove the theorem, we will first establish conditions under which $f^{0}$ is optimal that are very similar to the conditions under which a normal flow is optimal; specifically, we will show that $f^{0}$ is optimal if and only if the residual graph $T_{f^{0}}$ does not contain a certain negative-cost subgraph. We will then show a costpreserving correspondence between these subgraphs in $T_{f^{0}}$ and promenades in $G$, the Hamiltonian line graph on which Theorem 1 is based.

Agreeable Circulations. Suppose that $f$ is some feasible flow for a normal min-cost flow problem (without agreeability constraints) on an arbitrary graph $R$. Let $c[f]$ be the cost of $f$, and $R_{f}$ be the residual graph of $f$. A solution $f$ is optimal iff $R_{f}$ does not contain a negative-cost circulation.

Now consider the agreeable flow problem (RALP) on the trellis $T$, and the solution $f^{0}$ to RALP. Define the residual graph $T_{f^{0}}$ in the same manner as in normal min-cost flow. Now consider some other feasible agreeable flow $f^{\prime} \neq f^{0}$ in $T$. The circulation $f^{\prime}-f^{0}$ in $T_{f^{0}}$ has cost $c\left[f^{\prime}\right]-c\left[f^{0}\right]$. Since both $f^{0}$ and $f^{\prime}$ obey the agreeability constraints, the circulation $f^{\prime}-f$ also obeys the agreeability constraints. We call such a circulation an agreeable circulation.

Lemma 5 The RALP decoder succeeds if all agreeable circulations in $T_{f^{0}}$ have positive cost.

Proof: Suppose the RALP decoder fails, then $f^{0}$ is not the unique optimal solution to RALP. Let $f^{*} \neq f^{0}$ be some optimal solution to RALP. Consider the circulation $f^{\prime}=$ $f^{*}-f^{0}$. Since $c\left[f^{\prime}\right]=c\left[f^{*}\right]-c\left[f^{0}\right]$, and $c\left[f^{*}\right] \leqslant c\left[f^{0}\right]$, we know that $c\left[f^{\prime}\right] \leqslant 0$. It is clear that the sum or difference of two agreeable flows is agreeable, so $f^{\prime}$ is agreeable. 


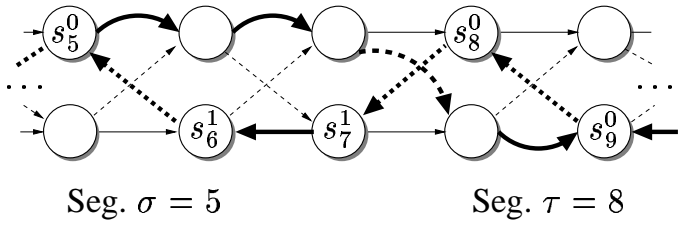

Figure 2. An example of a cycle $w(\sigma=5, \tau=8)$ in the residual graph $T_{f^{0}}$. The cycle (in bold) contains a subpath (straight bold lines) backwards along residual edges of $P_{x}$, where $P_{x}=\left(\ldots, s_{5}^{0}, s_{6}^{1}, s_{7}^{1}, s_{8}^{0}, s_{9}^{0}, \ldots\right)$. It also contains the complimentary forwards path from $s_{5}^{0}$ to $s_{9}^{0}$ (curved bold lines).

Lemma 6 The RALP decoder fails if there exists an agreeable circulation in $T_{f}$ with negative cost.

Proof: Let $f^{\prime}$ be the circulation in $T_{f^{0}}$ with negative cost. Let $f^{*}=f^{0}+f^{\prime}$. Since $c\left[f^{\prime}\right]<0, c\left[f^{*}\right]<c\left[f^{0}\right]$. By a similar argument as in Lemma $5, f^{*}$ is an agreeable flow. Since $f^{*}$ is a feasible solution to RALP with cost less than $f^{0}, f^{0}$ is not optimal, thus the RALP decoder fails.

Agreeable Circulations and Promenades. In the remainder of the proof, we show a correspondence between agreeable circulations in $T_{f^{0}}$ and promenades in $G$. We first define a special class of cycles in $T_{f^{0}}$ and show that the cost of a cycle in this class is the same as that of a corresponding subpath in $G$. Then we show that every simple cycle in $T_{f^{0}}$ is from this class. We conclude the proof by arguing that agreeable circulations always contain sets of these cycles that correspond to promenades in $G$ with the same cost.

Let $g(\sigma, \tau), \sigma<\tau$, be the path $\left(g_{\sigma}, g_{\sigma+1}, \ldots, g_{\tau}\right)$ of Hamiltonian edges in $G$. Now let $w(\sigma, \tau), \sigma<\tau$, be a certain cycle in $T_{f^{0}}$, as depicted in Figure 2 . We define $w(\sigma, \tau)$ formally as follows. Let $(\alpha[0]=0, \alpha[1], \ldots, \alpha[n]=0)$ be the sequence of states of the accumulator when encoding $x$ (note that $y_{t}=\alpha[t+1]$ ). For example, in Figure 2, $\alpha[\sigma]=0, \alpha[\sigma+1]=1, \alpha[\sigma+2]=1, \alpha[\tau]=0, \alpha[\tau+1]=$ 1. We have that $P_{x}=\left(s_{0}^{\alpha[0]}, s_{1}^{\alpha[1]}, \ldots, s_{n-1}^{\alpha[n-1]}, s_{n}^{\alpha[n]}\right)$, the path in $T$ taken by the encoder. In $T_{f^{0}}$, all edges along $P_{x}$ have a unit residual capacity going backwards towards $s_{0}^{0}$, since $f^{0}$ is the unit flow across path $P_{x}$. The cycle $w(\sigma, \tau)$ in $T$ consists of the backward subpath $\left(s_{(\tau+1)}^{\alpha[\tau+1]}, s_{\tau}^{\alpha[\tau]}, \ldots, s_{(\sigma+1)}^{\alpha[\sigma+1]}, s_{\sigma}^{\alpha[\sigma]}\right)$, and the forward subpath $\left(s_{\sigma}^{\alpha[\sigma]}, s_{\sigma+1}^{1-\alpha[\sigma+1]}, \ldots, s_{\tau}^{1-\alpha[\tau]}, s_{\tau+1}^{\alpha[\tau+1]}\right)$.

For $\tau>\sigma$, we let $g(\tau, \sigma)=g(\sigma, \tau)$ and $w(\tau, \sigma)=$ $w(\sigma, \tau)$. We will also use the notation $\lambda w$ to represent a circulation in $T_{f}$ that sends $\lambda$ units of flow around a residual cycle $w$. For a set $\mathcal{W}$ of cycles $w$ in $T_{f^{0}}$, we say that $\lambda \mathcal{W}$ is the circulation $\sum_{w \in \mathcal{W}} \lambda w$.

Claim $7 c[g(\sigma, \tau)]=c[w(\sigma, \tau)], 0 \leqslant \sigma<\tau<n$.
Proof: The cost of the cycle $w(\sigma, \tau)$ is the sum of the costs of its residual edges:

$$
\begin{aligned}
c[w(\sigma, \tau)]= & c\left[s_{\sigma}^{\alpha[\sigma]}, s_{\sigma+1}^{1-\alpha[\sigma+1]}\right] \\
& +\sum_{t=\sigma+1}^{\tau-1} c\left[s_{t}^{1-\alpha[t]}, s_{t+1}^{1-\alpha[t+1]}\right] \\
& +c\left[s_{\tau}^{1-\alpha[\tau]}, s_{\tau+1}^{\alpha[\tau+1]}\right] \\
& -\sum_{t=\sigma}^{\tau} c\left[s_{t}^{\alpha[t]}, s_{t+1}^{\alpha[t+1]}\right]
\end{aligned}
$$

Let $e$ be the indicator vector for errors occurring in the channel. In other words, for all $t, 0 \leqslant t<n$, let $e_{t}=1$ if $y_{t} \neq$ $v_{t}$, and $e_{t}=0$ otherwise. Note that every edge $\left(\cdot, s_{t+1}^{\alpha[t+1]}\right)$ in $T$ entering a node on $P_{x}$ has $c\left[\cdot, s_{t+1}^{\alpha[t+1]}\right]=e_{t}$. Similarly, every edge $\left(\cdot, s_{t+1}^{1-\alpha[t+1]}\right)$ has $c\left[\cdot, s_{t+1}^{1-\alpha[t+1]}\right]=1-e_{t}$. Thus we may conclude

$$
\begin{aligned}
c[w(\sigma, \tau)] & =\left(1-e_{\sigma}\right)+\left(\sum_{t=\sigma+1}^{\tau-1} 1-e_{t}\right)+e_{\tau}-\sum_{t=\sigma}^{\tau} e_{t} \\
& =\sum_{t=\sigma}^{\tau-1} 1-2 e_{t} \\
& =c[g(\sigma, \tau)] .
\end{aligned}
$$

Equation (4) follows from the definition of $G$.

Claim 8 For every simple cycle $C$ in $T_{f^{0}}, C=w(\sigma, \tau)$ for some $0 \leqslant \sigma<\tau<n$.

Proof: Let $s_{\tau}^{\alpha}$ be the node on the cycle $C$ that is the "closest" to $s_{n}^{0}$, i.e., $\tau$ is maximum among all nodes on $C$. The node $s_{\tau}^{\alpha}$ must be on $P_{x}$; otherwise, the outgoing edge from $s_{\tau}^{\alpha}$ on the cycle $C$ would go forwards in the trellis to some node $s_{\tau+1}^{\beta}$. Follow $C$ backwards along $P_{x}$ until it diverges from $P_{x}$ at some node $s_{\sigma}^{\gamma}$. The only remaining simple path from $s_{\sigma}^{\gamma}$ to $s_{\tau}^{\alpha}$ (that does not reuse an edge) is forward in the trellis along the edges that complete the cycle $w(\sigma, \tau)$.

Lemma 9 If there is a promenade in $G$ with negative cost, there is an agreeable circulation in $T_{f}$ with negative cost.

Proof: Let $M$ be a promenade in $G$ with negative cost. Let $1 / \lambda$ be the maximum number of occurrences of any single Hamiltonian edge in $M$. If the matching edges along $M$ are removed, what remains is a multiset of subpaths of the Hamiltonian path of $G$, of the form $g(\sigma, \tau)$. Let $\mathcal{U}=\left\{g\left(\sigma_{0}, \tau_{0}\right), \ldots, g\left(\sigma_{|\mathcal{U}|}, \tau_{|\mathcal{U}|}\right)\right\}$ be this set of subpaths, ordered by their occurrence during a traversal of $M$. In other words, one may traverse $M$ by following the path 
$g\left(\sigma_{0}, \tau_{0}\right)$, matching edge $\left(g_{\tau_{0}}, g_{\sigma_{1}}\right)$, path $g\left(\sigma_{1}, \tau_{1}\right), \ldots$, path $g\left(\sigma_{|\mathcal{U}|}, \tau_{\mathcal{U} \mid}\right)$, and finally matching edge $\left(g_{\tau_{|\mathcal{U}|}}, g_{\sigma_{0}}\right)$

Let $\mathcal{W}=\left\{w\left(\sigma_{0}, \tau_{0}\right), \ldots, w\left(\sigma_{|\mathcal{U}|}, \tau_{|\mathcal{U}|}\right)\right\}$ be the set of corresponding cycles in $T_{f^{\circ}}$. No edge in $\lambda \mathcal{W}$ has more than one unit of flow sent across it (by the definition of $\lambda$ ), so $\lambda \mathcal{W}$ is a feasible circulation in $T_{f^{0}}$.

Since all the matching edges of $G$ have zero cost, and $M$ has negative cost, we have $c[M]=\sum_{j=0}^{|\mathcal{U}|} c\left[g\left(\sigma_{j}, \tau_{j}\right)\right]<0$. Therefore by Claim $7, \sum_{j=0}^{|\mathcal{W}|} c\left[w\left(\sigma_{j}, \tau_{j}\right)\right]<0$. It follows that $c[\lambda \mathcal{W}]<0$.

It remains to show that $\lambda \mathcal{W}$ is agreeable. Consider the first cycle in $\mathcal{W}$, namely $w\left(\sigma_{0}, \tau_{0}\right)$. As Figure 2 shows, the only agreeability constraints violated by sending flow around this cycle are at trellis segments $\sigma_{0}$ and $\tau_{0}$; all segments in-between have the same amount of flow on switchedges as they did before.

Assume wlog that $P_{x}$ has a remain-edge at segment $\tau_{0}$, so $\lambda w\left(\sigma_{0}, \tau_{0}\right)$ increases the flow on switch-edges by $\lambda$ at segment $\tau_{0}$. Since $\left(g_{\tau_{0}}, g_{\sigma_{1}}\right)$ is a matching edge (by definition of $\mathcal{U}),\left\{\tau_{0}, \sigma_{1}\right\} \in \mathcal{X}$. Therefore $P_{x}$ must obey the agreeability constraint at segments $\tau_{0}$ and $\sigma_{1}$; in other words, it must also have a remain-edge at segment $\sigma_{1}$. It follows that $\lambda w\left(\sigma_{1}, \tau_{1}\right)$ increases the flow on switchedges by $\lambda$ at segment $\sigma_{1}$. Therefore the agreeability constraint for information bit $x_{i}$ is preserved by the circulation $w\left(\sigma_{0}, \tau_{0}\right)+w\left(\sigma_{1}, \tau_{1}\right)$, and the only violated agreeability constraints are at segments $\sigma_{0}$ and $\tau_{1}$. A symmetric argument holds if $P_{x}$ has a switch-edge at segment $\tau_{0}$.

By repeatedly applying the same argument along $\mathcal{W}$, we may conclude that the circulation $\lambda \mathcal{W}$ only violates agreeability constraints at $\sigma_{0}$ and $\tau_{|\mathcal{W}|}$. Since $\left\{\sigma_{0}, \tau_{|\mathcal{W}|}\right\} \in \mathcal{X}$, we have that $\lambda \mathcal{W}$ does not violate any agreeability constraints.

Lemma 10 If all promenades in $G$ have positive cost, all agreeable circulations in $T_{f^{\circ}}$ have positive cost.

Proof: Suppose $f^{\prime}$ is an agreeable circulation in $T_{f^{0}}$ where $c\left[f^{\prime}\right]<0$. Let $\lambda$ be the gcd of all the values of flow on edges of $f^{\prime}$. Consider the cycle decomposition of $f^{\prime}$ where every cycle has $\lambda$ units of flow around it. Let $\mathcal{W}$ be the collection of cycles in this decomposition. By claim 8 , every cycle in $T_{f^{0}}$ is of the form $w(\sigma, \tau)$. For all $t, 0 \leqslant t<n$, let $B_{t}=\{w(\sigma, \tau) \in \mathcal{W}: \sigma=t$ or $\tau=t\}$.

For some set $A$ of cycles $w(\sigma, \tau)$, we use the notation $f \in A$ to denote the flow $\lambda w(\sigma, \tau)$ for some arbitrary cycle $w(\sigma, \tau) \in A$.

Consider some $X_{i}=\{t, \hat{t}\}$. Let $z_{t}=f^{\prime}\left(s_{t}^{0}, s_{t+1}^{1}\right)+$ $f^{\prime}\left(s_{t}^{1}, s_{t+1}^{0}\right)$. Because each cycle $w(\sigma, \tau) \in \mathcal{W}$ only affects the agreeability constraints at segments $\sigma$ and $\tau, z_{t}=$ $\sum_{f \in B_{t}} \lambda f\left(s_{t}^{0}, s_{t+1}^{1}\right)+\lambda f\left(s_{t}^{1}, s_{t+1}^{0}\right)$. If $x_{i}=0$, then for all $f \in B_{t}, f$ has $\lambda$ units of flow on one of the edges $\left(s_{t}^{0}, s_{t+1}^{1}\right)$ or $\left(s_{t}^{0}, s_{t+1}^{1}\right)$, and zero units on the other one. Thus $f$ will add $\lambda$ to $z_{t}$ in the above sum. If $x_{i}=1$, then $f$ has $-\lambda$ flow on either $\left(s_{t}^{0}, s_{t+1}^{1}\right)$ or $\left(s_{t}^{0}, s_{t+1}^{1}\right)$, and contributes $-\lambda$ to the sum. Therefore,

$$
z_{t}= \begin{cases}\lambda\left|B_{t}\right| & \text { if } x_{i}=0 \\ -\lambda\left|B_{t}\right| & \text { if } x_{i}=1\end{cases}
$$

Define $z_{\hat{t}}$ similarly. By the same logic,

$$
z_{\hat{t}}= \begin{cases}\lambda\left|B_{\hat{t}}\right| & \text { if } x_{i}=0 \\ -\lambda\left|B_{\hat{t}}\right| & \text { if } x_{i}=1\end{cases}
$$

Since $f^{\prime}$ is agreeable, $z_{t}=z_{\hat{t}}$. Thus we may conclude that $\left|B_{t}\right|=\left|B_{\hat{t}}\right|$. For all $\{t, \hat{t}\} \in \mathcal{X}$, create a one-to-one correspondence between the members of $B_{t}$ and $B_{\hat{t}}$ (we can do this because the sets are the same size).

Create an auxiliary multigraph $H$ with a node $v[w(\sigma, \tau)]$ for each $w(\sigma, \tau) \in \mathcal{W}$. Add edges according to the correspondence we just created for each $\{t, \hat{t}\} \in \mathcal{X}$. Note that if $w(t, \hat{t}) \in \mathcal{W}$, it would be in both $B_{t}$ and $B_{\hat{t}}$. In this case, the correspondence may assign $v[w(t, \hat{t})]$ to itself; we represent this by a self-loop on $v[w(t, \hat{t})]$.

Each $w(\sigma, \tau) \in \mathcal{W}$ is in exactly two sets: $B_{\sigma}$ and $B_{\tau}$. Therefore, $H$ is 2-regular, a collection $\mathcal{Y}$ of simple cycles (where a node with a self-loop is considered a cycle). For a cycle $Y \in \mathcal{Y}$, let $\mathcal{W}_{Y}=\{w(\sigma, \tau) \in \mathcal{W}$ : $v[w(\sigma, \tau)] \in Y\}$. The set $\left\{\mathcal{W}_{Y}: Y \in \mathcal{Y}\right\}$ constitutes a partition of $\mathcal{W}$ into subsets, so $f^{\prime}=\sum_{f \in \mathcal{W}} f=$ $\sum_{Y \in \mathcal{Y}} \sum_{f \in \mathcal{W}_{Y}} f$. Since $c\left[f^{\prime}\right]<0$, there must be some $Y^{*} \in \mathcal{Y}$ such that $\sum_{f \in \mathcal{W}_{Y^{*}}} c[f]<0$. It follows that $\sum_{w(\sigma, \tau) \in \mathcal{W}_{Y^{*}}} c[w(\sigma, \tau)]<0$.

We build a promenade $M$ in $G$ by following the cycle $Y$. We begin with an arbitrary node $v\left[w\left(\sigma_{0}, \tau_{0}\right)\right]$, and add the edges of the path $g\left(\sigma_{0}, \tau_{0}\right)$ to $M$. We then follow an edge in $H$ to a node $v\left[w\left(\sigma_{1}, \tau_{1}\right)\right]$, where $\left\{\tau_{0}, \sigma_{1}\right\} \in \mathcal{X}$, by definition of $H$. When we follow this edge, we add the matching edge $\left(\tau_{0}, \sigma_{1}\right)$ to $M$, then the path $g\left(\sigma_{1}, \tau_{1}\right)$. We continue this way until we complete the cycle $Y$, and thus close the promenade $M$.

Let $\mathcal{U}$ be the set of subpaths $g(\sigma, \tau)$ we added to $M$ while following $Y$. Matching edges have zero cost, so $c[M]=$ $\sum_{g(\sigma, \tau) \in \mathcal{U}} c[g(\sigma, \tau)]$. Since $\sum_{w(\sigma, \tau) \in \mathcal{W}_{Y^{*}}} c[w(\sigma, \tau)]<0$, we have $c[M]=\sum_{g(\sigma, \tau) \in \mathcal{U}} c[g(\sigma, \tau)]<0$ by Claim 7 . Thus $M$ is a negative-cost promenade, and we have a contradiction.

Theorem 1 is implied by Lemmas 5, 6, 9 and 10 .

\section{Generalization to Concatenated Codes}

In this section we give a generic LP to decode a repetition code concatenated with any other inner rate- 1 code, with an interleaver between them. The LP can be generalized further to apply to any set of parallel or serially concatenated codes of any rate, connected by interleavers. We defer this 
general form to a later version, though it is not difficult to derive.

We model the inner code using a simple graph (trellis) that represents each input string by a path from a start node to an end node. Any rate- 1 binary code can be modeled this way, though for some codes, the trellis could require exponential size. So, the LP is only of polynomial size if the code can be described by a polynomial-sized trellis. Any convolutional code (see [19]) has a simple linear-size trellis.

Let trellis $T$ be a directed graph with the following properties: (i) There is a specified start node $s_{0}$. For all other nodes $s$ in $T$, all paths from $s_{0}$ to $s$ have equal length. Let $L_{t}$ be the set of nodes distance $t$ from $s_{0}$. (ii) Nodes in $L_{n}$ have no outgoing edges. All other nodes have two outgoing edges: an "input-0" edge, and an "input-1" edge. Let $S_{t}$ be the set of input-1 edges leaving nodes in $L_{t}$. (iii) Each edge is labeled with a code bit 0 or 1 .

Let $\pi$ be a permutation $\pi_{j}:\{0, \ldots, n-1\} \rightarrow$ $\{0, \ldots, n-1\}$. The code $(T, \pi)$ encodes an information word $x$ of length $k$ as follows. (i) Let $x^{\prime}$ be a binary string of length $n$, where $x_{t}^{\prime}=x_{\left\lfloor\pi^{-1}(t) / \ell\right\rfloor}$. (ii) From the start node $s_{0}$ of $T$, follow a path in $T$ using bits from $x^{\prime}$ : on step $t$ of the path, follow the "input- 0 " edge if $x_{t}^{\prime}=0$, and follow the "input-1" edge if $x_{t}^{\prime}=1$. Concatenate the labels on the edges of the path to obtain a code word $y$ of length $n$.

We define the linear program TCLP as follows. As in RALP, we have a flow varaible $f(e)$ for each edge in the trellis $T$. We also have free variables $z_{i}$ for each information bit $x_{i}$. The cost $c[e]$ of an edge $e$ entering a node from $L_{t}$ is the Hamming distance between the label on the edge and the received bit $v_{t}$. For each node $s$ in $T$, define $\delta(s)$ to be the set of outgoing edges from $s$, and $\gamma(s)$ to be the set of incoming edges. For all $i \in\{0, \ldots, k-1\}$, let $X_{i}$ be the set of indicies to which information bit $x_{i}$ was repeated and permuted, i.e., $X_{i}=\{\pi(\ell i), \pi(\ell i+1), \ldots, \pi(\ell i+\ell-1)\}$. Let $\mathcal{X}=\left\{X_{i}: i \in\{0, \ldots, k-1\}\right\}$.

$$
\begin{array}{cl}
\text { TCLP: } & \min \sum_{e \in T} c[e] f(e) \quad \text { s.t. } \\
\sum_{e \in \delta\left(s_{0}\right)} f(e)=1 & \\
\sum_{e \in \gamma(s)} f(e)=\sum_{e \in \delta(s)} f(e) \quad \forall s \in T, s \neq s_{0}, s \notin L_{n} \\
\sum_{e \in S_{t}} f(e)=z_{i} \quad \forall X_{i} \in \mathcal{X}, t \in X_{i} \\
0 \leqslant f(e) \leqslant 1 \quad \forall e \in T
\end{array}
$$

A decoder based on TCLP has the ML certificate property for the same reasons the RALP decoder did. It is also not hard to derive a generalized "promenade" structure for
TCLP, and prove a theorem analogous to Theorem 1 for this class of codes. The challenge then is to prove that for some interleaver, it is unlikely that there will be a "promenade" with cost less than or equal to zero. It would be interesting to see a general construction to derive interleavers for this class of codes.

\section{Future Work}

Improving the Running Time The obvious drawback of our approach to decoding is the complexity of solving a linear program. Even though interior point methods run in polynomial-time (and the simplex algorithm often faster), most applications of error-correcting codes require a more efficient decoding algorithm. There are two possible solutions to this problem, and both have some important unanswered questions.

The first option is to try and solve RALP or TCLP combinatorially. For the case of $\mathrm{RA}(2)$ codes, the resulting agreeable flow problem can be reduced to an instance of normal min-cost flow, and thus yields a more efficient combinatorial algorithm. We leave the details for a later version. It is an interesting open question as to whether combinatorial solutions exist for $\mathrm{RA}(\ell), \ell \geqslant 3$, or other codes.

The agreeable flow problem has a more general formulation that could apply to areas outside of coding theory. We define the min-cost agreeable flow problem as follows:

Min-Cost Agreeable Flow: Given a directed network $G=(V, E)$, a source $s \in V$ and a sink $t \in V$ with a demand $\alpha$, capacities $u: E \rightarrow \mathbb{R}^{+}$and costs $c: E \rightarrow \mathbb{R}$ on the edges, and a sequence of edge sets $\mathcal{A}=\left\{A_{1}, \hat{A}_{1}, A_{2}, \hat{A}_{2}, \ldots, A_{m}, \hat{A}_{m}\right\}$, find a minimum-cost $\alpha$-unit flow $f$ from $s$ to $t$, where $\forall\left(A_{i}, \hat{A}_{i}\right)$, the total flow going through arcs in $A_{i}$ is equal to the total flow going through $\operatorname{arcs}$ in $\hat{A}_{i}$.

Any LP with a constraint matrix made up of $\{+1,-1\}$ can be expressed using only the agreeability constraints of the above formulation, so we would not expect to be able to solve Min-Cost Agreeable Flow combinatorially in its full generality. However, the specialized structure of RALP or TCLP may allow a combinatorial solution. In general, it is an interesting question to determine how the min-cost agreeable flow problem must be restricted in order to make it solvable combinatorially.

The second option for improving efficiency is to use an iterative decoder. We showed (with Wainwright [8]) that the iterative TRMP algorithm of Wainwright, Jaakkola and Willsky [18], when applied to the problem of decoding turbo-like codes, outputs exactly the solution to TCLP. We have done some testing of this algorithm applied to various 
codes, and it seems to converge reasonably well. However, it is not known whether this algorithm converges in general, or how long it takes to converge when it does.

Improving the Error Bounds. The RA code with rate $1 / 2-o(1)$ that we were able to analyze completely is not the best code experimentally in the literature. We are currently trying to understand the combinatorics behind more complicated codes such as a rate 1/3 RA code, and the classic turbo code (parallel concatenated convolutional code). In order to provide better bounds for these codes, we need to prove that negative-cost "promenade"-like subgraphs are unlikely. Theorem 1 suggested a design for an interleaver for the rate $1 / 2-o(1) \mathrm{RA}$ code. It would be interesting to see if other design suggestions can be derived for more complex turbo-like codes.

Interpreting Iterative Decoders as Optimization Algorithms. We have already begun the work of connecting the world of iterative decoders to our LP decoder [8]. However, preliminary testing suggests that BP algorithms outperform our LP decoder. The reason for this difference is not well understood. If we were able to interpret standard BP decoders as global optimization routines, perhaps we would be able to bound their error rate using the same techniques.

Using the Polytope for ML Decoding. We observed through experimentation that the integrality gap of TCLP applied to RA codes was usually very good, and so the solution to TCLP provided a good lower bound for use in a branch-and-bound search for the ML code word. Although not guaranteed to run in polynomial time, our branch-andbound search finished much sooner than a naive ML decoding algorithm would have. More details of these experiments will appear in a later version.

More applications of LP-based decoding. Our analysis of the error probability is over the random coin flips of the channel; in the context of TCLP, this translates to the probability, in a fixed polytope, over a random direction of the objective function, that the LP solution was integral. It would be interesting to write LPs for other codes (such as lowdensity parity check codes or expander-based codes) and apply the same sort of analysis. Perhaps this sort of analysis could have other applications outside of coding theory.

\section{Acknowledgements}

We would like to thank Piotr Indyk, Martin Wainwright and Matthias Ruhl for helpful discussions. We also thank Dan Spielman, David Forney, Ibrahim Abou-Faycal, Matteo Frigo, Vanu Bose, John Chapin, Andrew Russell, Madhu Sudan, Ryan O'Donnell, Muriel Medard and Matt Levine for their comments, suggestions, and support.

\section{References}

[1] R. K. Ahuja, T. L. Magnanti, and J. B. Orlin. Network Flows. Prentice-Hall, 1993.

[2] L. Bazzi, M. Mahdian, S. Miller, and D. Spielman. The minimum distance of turbo-like codes. manuscript, 2001.

[3] C. Berrou, A. Glavieux, and P. Thitimajshima. Near Shannon limit error-correcting coding and decoding: turbocodes. Proc. IEEE International Conference on Communication (ICC), Geneva, Switzerland, pages 1064-1070, May 1993.

[4] N. Biggs. Constructions for cubic graphs with large girth. Electronic Journal of Combinatorics, 5(A1), 1998.

[5] M. Breiling and J. Huber. Upper bound on the minimum distance of turbo codes using a combinatorial approach, 2001.

[6] D. Divsalar, H. Jin, and R. McEliece. Coding theorems for 'turbo-like' codes. Proc. 36th Annual Allerton Conference on Comm., Control, and Computing, pages 201-210, Sept 1998.

[7] P. Erdös and H. Sachs. Regulare graphen gegebene taillenweite mit minimaler knotenzahl. Wiss. Z. Univ. Hall Martin Luther Univ. Halle-Wittenberg Math.-Natur.Reine, 12:251-257, 1963.

[8] J. Feldman, D. Karger, and M. Wainwright. Linear programming based decoding of turbo-like codes and its relation to iterative approaches. Proc. 40th Annual Allerton Conference on Comm., Control, and Computing, October 2002.

[9] G. Forney. Convolutional codes II: Maximum likelihood decoding. Inform. Control, 25:222-266, 1974.

[10] N. Kahale and R. Urbanke. On the minimum distance of parallel and serially concatenated codes. IEEE International Symposium on Information Theory, 1998.

[11] F. MacWilliams and N. Sloane. The Theory of Error Correcting Codes. North-Holland, 1981.

[12] R. McEliece, D.MacKay, and J. Cheng. Turbo decoding as an instance of Pearl's belief propagation algorithm. IEEE Journal on Selected Areas in Communications, 16(2):140 $152,1998$.

[13] T. Richardson, M. A. Shokrollahi, and R. L. Urbanke. Design of capacity-approaching irregular low-density paritycheck codes. Information Theory, IEEE Transactions on, 47(2):619-637, 2001.

[14] T. Richardson and R. Urbanke. The capacity of low-density parity-check codes under message-passing decoding. IEEE Transactions on Information Theory, 47(2), February 2001.

[15] N. Sauer. Extremaleigenschaften regularer graphen gegebener taillenweite, iandii. Sitzungsberichte Osterreich. Acad. Wiss. Math. Natur. Kl., S-BII(176):27-43, 1967.

[16] A. Viterbi. Error bounds for convolutional codes and an asymptotically optimum decoding algorithm. IEEE Trans. Inform. Theory, IT-13:260-269, Apr. 1967.

[17] B. Vucetic and J. Yuan. Turbo Codes. Kluwer Academic Publishers, Dordrecht, The Netherlands, 2000.

[18] M. J. Wainwright, T. S. Jaakkola, and A. S. Willsky. MAP estimation via agreement on (hyper)trees: Message-passing and linear programming approaches. MIT LIDS Tech. report, July 2002.

[19] S. Wicker. Error Control Systems for Digital Communication and Storage. Prentice-Hall, Englewood Cliffs, NJ, 1995. 